\title{
Article
}

\section{Vitamin D deficiency in traumatic brain injury and its relationship with severity of injury and quality of life: a prospective, observational study}

Toman, Emma, Bishop, JR, Davies, DJ, Su, Z, Criseno, S, Mason, A, Toogood, A and Belli, A

Available at http://clok.uclan.ac.uk/21589/

Toman, Emma, Bishop, JR, Davies, DJ, Su, Z, Criseno, S, Mason, A, Toogood, A and Belli, A (2017) Vitamin D deficiency in traumatic brain injury and its relationship with severity of injury and quality of life: a prospective, observational study. Journal of Neurotrauma, 34 (7). pp. 1448-1456. ISSN 0897-7151

It is advisable to refer to the publisher's version if you intend to cite from the work. http://dx.doi.org/10.1089/neu.2016.4494

For more information about UCLan's research in this area go to http://www.uclan.ac.uk/researchgroups/ and search for <name of research Group>.

For information about Research generally at UCLan please go to http://www.uclan.ac.uk/research/

All outputs in CLoK are protected by Intellectual Property Rights law, including Copyright law. Copyright, IPR and Moral Rights for the works on this site are retained by the individual authors and/or other copyright owners. Terms and conditions for use of this material are defined in the policies page. 


\title{
Vitamin $D$ deficiency in traumatic brain injury and its relationship with severity of injury and quality of life: a prospective, observational study
}

\author{
Dr Emma Toman MBChB (Corresponding Author) \\ Neurosurgery Clinical Research Fellow, National Institute for Health Research Surgical Reconstruction and Microbiology \\ Research Centre (NIHR SRMRC), Queen Elizabeth Hospital, Edgbaston, Birmingham, United Kingdom B15 2 WB. \\ Email - Emma.Toman@nhs.net \\ Tel - +44(0)1213716740
}

Dr Jonathon R. B. Bishop PhD

Medical Statistician, NIHR SRMRC, Public Health Building, University of Birmingham, Edgbaston, United Kingdom B15 2TT.

Email - J.Bishop.1@bham.ac.uk

Tel - +44 (0)1213716740

Mr David J. Davies MB BCh MRCS(Eng)

Neurosurgery Clinical Research Fellow, NIHR SRMRC, Queen Elizabeth Hospital, Edgbaston, Birmingham, United Kingdom B15 2WB.

Email - David.Davies@uhb.nhs.uk

Tel - +44 (0)1213716741

Dr Zhangjie Su MBBS, MSc, PhD

Neurosurgery Junior Specialist Doctor, Neurosurgery Department, Queen Elizabeth Hospital, Edgbaston, Birmingham, United Kingdom B15 2WB.

Email - Zhangjie.Su@uhb.nhs.uk

Tel - +44 (0)1213716740

Mr Sherwin Criseno, BSc, PG Dip (Education)

Lead Clinical Specialist Nurse Endocrinology, Endocrinology Department, Queen Elizabeth Hospital, Edgbaston, Birmingham, United Kingdom B15 2WB.

Email - Sherwin.Criseno@uhb.nhs.uk

Tel - +44 (0)1213716950

Ms Andrea Mason BSc

Clinical Nurse Specialist Endocrinology, Endocrinology Department, Queen Elizabeth Hospital, Edgbaston, Birmingham, United Kingdom B15 2WB.

Email - Andrea.Mason@uhb.nhs.uk

Tel - +44 (0)1213716950

Dr Andrew A. Toogood MBChB, MRCP, MD, FRCP

Consultant Endocrinologist, Endocrinology Department, Queen Elizabeth Hospital, Edgbaston, Birmingham, United Kingdom B15 2WB.

Email - Andrew.Toogood@uhb.nhs.uk

Tel - +44(0)1213712000

Prof Antonio Belli MD FRCS (Gla), FRCS (Surgical Neurology)

Professor of Neurological Trauma and Consultant Neurosurgeon. NIHR SRMRC, Institute of Inflammation and Ageing, College of Medical and Dental Sciences, IBR Building, University of Birmingham, Edgbaston, Birmingham, United Kingdom, B15 2TT.

Email - A.Belli@bham.ac.uk

Tel - +44 (0)1213716740 
Word count (minus abstract, tables, figures and references): 3481 


\section{ABSTRACT}

This single-centre prospective observational study aims to describe the prevalence of vitamin D deficiency (VDD) in the traumatic brain injury (TBI) population and identify any relationship between vitamin $D$ and severity of head injury or quality of life.

$124 \mathrm{TBI}$ patients had serum vitamin $\mathrm{D}(25-\mathrm{OHD})$ levels measured at the local post-TBI endocrine screening clinic over 20 months. Quality of Life after Brain Injury (QOLIBRI) questionnaires were completed by the patient concurrently. A multivariate regressional analysis was performed, controlling for age, season, ethnicity, time since injury, TBI severity and gender.

$34 \%(n=42)$ of the cohort were vitamin D deficient $(25-\mathrm{OHD}<25 \mathrm{nmol} / \mathrm{L})$ with a further $23 \%(n=29)$ having insufficient levels $(25-\mathrm{OHD} 25-50 \mathrm{nmol} / \mathrm{L})$. Vitamin $\mathrm{D}$ was significantly lower in severe TBI compared to mild TBI ( $n=95, p=0.03, C I 95 \%-23.60$ to -1.21 , mean effect size $12.40 \mathrm{nmol} / \mathrm{L})$. There was a trend for self-reported quality of life to be better in patients with optimum vitamin $D$ levels compared to patients with deficient vitamin $D$ levels, controlling for severity of injury $(n=81, p=0.05, \mathrm{Cl}$ $95 \%-0.07$ to 21.27$)$.

This is the first study to identify a significant relationship between vitamin $D$ levels and severity of head injury. Clinicians should actively screen for and treat VDD in head injured patients to reduce the risk of further morbidity such as osteomalacia and cardiovascular disease. Future research should establish the natural history of vitamin D levels following TBI to identify at which stage VDD develops and whether vitamin $\mathrm{D}$ replacement could have a beneficial effect on recovery and quality of life.

Key words: traumatic brain injury, vitamin D, vitamin D deficiency, neurotrauma, endocrinology 


\section{INTRODUCTION}

Traumatic brain injury (TBI) is the most common cause of mortality and disability among young people in high-income countries, and worldwide the incidence of TBI is increasing.(1) It is becoming increasingly obvious that in addition to the acute costs involved in caring for these patients we must also consider the significant of cost chronic sequelae including physical disability, neuropsychological disturbance and biochemical dysfunction.

Although vitamin $D$ is technically mis-named and is in-fact a secosteroid, it is still grouped with other vitamins for historical reasons. The main source of vitamin D for humans is sunlight. Ultraviolet B light causes the photolysis of 7-dehydrocholesterol to previtamin D in the skin.(2) Previtamin D undergoes isomerisation producing vitamin $\mathrm{D}_{3}$ and is then metabolised twice; first in the hepatic mitochondria to form 25-hydroxyvitamin $\mathrm{D}_{3}(25-\mathrm{OHD})$ and then in renal mitochondria to form the active vitamin $\mathrm{D}$ molecule 1,25 hydroxyvitamin $D_{3}\left(1,25-(\mathrm{OH})_{2} \mathrm{D}_{3}\right)$ (see figure 1).(3) The second source of vitamin $D$ for humans comes from dietary intake. UK food sources of vitamin D include oily fish, eggs and fortified fat spreads and cereals.(4) In clinical practice we measure $25-\mathrm{OHD}$ serum levels as $1,25-(\mathrm{OH})_{2} \mathrm{D}_{3}$ circulates in such a low concentration that assessing levels becomes challenging.

Given the emerging evidence supporting its role in an increasing number of complex processes vitamin $\mathrm{D}$ has become a hot topic; from discussion on online forums(5) to governmental guidelines. In 2014 the English National Institute for Health and Care Excellence (NICE) published guidelines with the aim to combat VDD across the general population.(6) They identified the following "at risk" groups:

1. infants and children aged under 5 years

2. pregnant and breastfeeding women

3. people over 65 years

4. low sunlight exposure including those who cover their skin for cultural reasons or are confined to indoors for long periods

5. people with darker skin e.g. those of African, African-Caribbean or South Asian family $\operatorname{origin}(6)$ 
An English National Diet and Nutrition Survey was undertaken from 2008 to 2012 and results published in 2014. On average, intake of vitamins was adequate, excluding vitamin D.(7) In addition to nutritional questionnaires serum was sampled from participants. Overall, the survey reported a prevalence of VDD in $22.8 \%$ of adults aged $19-64$ years with $21.7 \%$ prevalence in males and $24.0 \%$ prevalence in females (see table 1).

Vitamin D deficiency contributes to a myriad of diseases and is a risk factor for conditions with significant morbidity. VDD is most commonly known as a cause of rickets in children and osteomalacia in adults. Complications include muscle weakness, skeletal pain, increased fracture risk and skeletal deformities such as "bowlegs" and thinning of the skull; "craniotabes".(8) Vitamin D has also been found to have a complex modulating action within the immune system,(9-11) has been linked with cardiovascular disease,(12) is known to play a role in multiple cancers(13) and has been widely investigated in other conditions such as multiple sclerosis, type 1 and 2 diabetes mellitus, preeclampsia, rheumatoid arthritis, dementia and metabolic syndrome.(14, 15)

Within the context of clinical TBI management, endocrinological deficiency and pituitary malfunction are becoming more familiar as longer term post-injury sequelae with pituitary dysfunction and diabetes insipidus demonstrating a prevalence of $5.4 \%(16)$ to $19 \%(17)$ and $2.9 \%(18)$ respectively. To detect these conditions, our unit developed a collaborative post-TBI endocrine screening clinic, operational since July 2013. In addition to screening for pituitary dysfunction, serum vitamin D levels were also measured following a published study demonstrating vitamin D supplementation to significantly reduce the incidence of seizures.(19) It appeared that a large proportion of patients screened were found to be either vitamin $\mathrm{D}$ insufficient or deficient. This led our team to generate the hypothesis that VDD was more prevalent in TBI patients compared to the general population. Given the emerging evidence that vitamin D plays important roles within the brain we also hypothesised that vitamin $D$ levels decrease as severity of injury increases.

This paper aims to describe the prevalence of vitamin D deficiency (VDD) in the post-TBI population and quantify the relationship between vitamin $D$ levels and severity of head injury or subsequent quality of life (QoL). 


\section{METHODS}

The Queen Elizabeth Hospital Birmingham (QEHB) has a well-established post-TBI endocrine screening outpatient clinic. Patients were referred here directly from the consultant lead-neurosurgical TBI clinic for anterior pituitary screening and vitamin D (25-OHD) level testing.

As part of the TBI clinic attendance patients also completed the Quality of Life after Brain Injury (QOLIBRI) questionnaire. The QOLIBRI is an internationally recognised tool specifically designed for TBI patients.(20) Self-reported scores given by the patient generate a score on a scale from 0-100 where 0 is the lowest QoL and 100 is the best QoL.

Details of 124 patients referred for screening were collected between the dates of September 2013 to July 2015. Demographic, clinical and laboratory data were collected from the hospital IT systems. Severity of head injury was categorised by best immediately post-resuscitative GCS, separated into mild (GCS 13-15), moderate (GCS 9-12) and severe (3-8). As some patients are referred to the TBI clinic from local hospitals post-resuscitative GCS data were not always available. The UK seasons were categorised as spring (March to May), summer (June to August), autumn (September to November) and winter (December to February). There was a wide variability in the time periods between injury and sampling across the cohort. To explore whether this plays a significant role in levels of vitamin D, "time since injury" was included as a variable in multivariate analysis. Six months is a widely accepted outcome milestone in TBI and is a broadly used across TBI academia. It is for this reason the authors chose 6 months as the cut-off point.

The English Scientific Advisory Committee on Nutrition released a draft report on vitamin D in 2015. The report described various reference ranges that have been quoted in the literature, many of which vary.(21) The general consensus on the level considered deficient is $25-\mathrm{OHD}<25 \mathrm{nmol} / \mathrm{L}$ and this is the cut-off used by NICE guidance on vitamin D.(6) Additional reference ranges we have used in this study (see table 2) are comparable to recent published studies although we must stress there is no internationally agreed reference range for vitamin D.

25-OHD was measured by the University Hospitals Birmingham laboratories using the gold standard, liquid chromatography-tandem mass spectrometry. 


\section{Statistical analysis}

The statistical software "R" was used to perform these analyses and cut-off for clinical significance set at $p<0.05$. Multiple multivariate regressional analyses were performed with vitamin $D$ treated as both categorical and continuous data. All statistical analyses were performed by a University affiliated medical statistician.

\section{Exclusions criteria}

Exclusion criteria included any patient with renal or hepatic failure and any receiving vitamin D supplementation at the time of sampling. Liver and kidney function were assessed simultaneously by serum testing and a drugs history documented. 


\section{RESULTS}

One-hundred and twenty-four clinic patients were included in this study from the dates September 2013 - July 2015. No patients were excluded. The median age was 41 years (range 16-90) and 79\% were male. The median time between injury and vitamin D sampling was 285 days (range 32-939 days).

Twenty-three percent of the cohort had a severe TBI, $14 \%$ moderate and $63 \%$ mild. With regards time of sampling; $27 \%(n=33)$ were taken in the spring, $20 \%(n=25)$ in summer, $27 \%(n=34)$ in autumn and $26 \%(n=32)$ in the winter. Ethnicity was broken down to Caucasian $73 \%(n=91)$, South Asian $10 \%$ $(n=12)$ and other/unspecified $17 \%(n=21)$.

Overall $34 \%(n=42)$ of the cohort was vitamin D deficient with a further $23 \%(n=29)$ demonstrating vitamin $\mathrm{D}$ insufficiency. Table 3 provides detailed breakdown of prevalence within the different groups. The group with the highest percentage of severe TBls was males aged 19-64 years at $28.0 \%(n=23)$. VDD was present in $48.3 \%$ of severe TBI patients compared to $29.4 \%$ in moderate and $26.5 \%$ in mild (see table 4).

Excluding TBI severity data a multivariable linear regression was performed across all 124 patients. Vitamin $\mathrm{D}$ was treated as a continuous variable and was modelled by age, ethnicity, season, time since injury $<6 \mathrm{~m}$ and gender (see table 5 ). This analysis demonstrated that levels of vitamin $\mathrm{D}$ taken in the summer and autumn were significantly higher than samples taken in the winter. Regarding significant findings in ethnicity, mean vitamin $D$ was found to be $17.68 \mathrm{nmol} / \mathrm{L}$ higher in Caucasians when compared to South Asians ( $p=0.04, \mathrm{Cl} 95 \% 0.50$ to 34.76). No significant difference in vitamin D was demonstrated between samples taken within 6 months from injury and samples taken over 6 months from injury ( $p=0.44, \mathrm{Cl} 95 \%-15.30$ to 6.65$)$. No significant differences in vitamin $\mathrm{D}$ were found with change in age or gender (see table 5 and figure 2).

TBI severity was then included in the multivariate linear regression controlling again for age, ethnicity, season, time since injury $<6 \mathrm{~m}$ and gender. This was a separate analysis as only 95 patients had TBI severity data available. Vitamin D was significantly lower in severe TBI compared to mild TBI $(p=0.03$, 
CI $95 \%-23.60$ to -1.21 ) with mean vitamin D in severe TBI $12.40 \mathrm{nmol} / \mathrm{L}$ lower than in mild TBI (see table 6 and figure 3).

To analyse QoL, QOLIBRI score was modelled by vitamin D as a categorical variable in a univariate linear regression and controlled for TBI severity. This analysis incorporated 81 observations patients as QOLIBRI questionnaires were not completed for all patients. Vitamin D category "deficient" was used as the reference. QOLIBRI was demonstrated to be increased in the patient cohort with optimal vitamin $D$ levels compared with those with deficient vitamin $D$ levels, although this finding does not quite reach significance ( $p=0.05, \mathrm{Cl} 95 \%-0.07$ to 21.27 ). Patients with optimal vitamin $\mathrm{D}$ scored 10.60 points higher on the QOLIBRI scale compared to the deficient category. No significant difference in QOLIBRI scores were found between the vitamin D deficient group in comparison with insufficient or adequate vitamin $D$ cohorts (see table 7 , figure 4 ). When vitamin $D$ is treated as a continuous variable, the correlation between vitamin D levels and QOLIBRI score is $0.165(95 \% \mathrm{Cl}-0.026$ to 0.344), which suggests a weak relationship between these variables (Fig 5). As vitamin D levels increase, there is a weak association with increased QOLIBRI score $(p=0.09)$ but the magnitude of this is small. 


\section{DISCUSSION}

Overall the prevalence of VDD in the TBI is increased compared to the English national prevalence and the local prevalence of $24 \%$ reported in inner-city Birmingham outpatient services.(22) There are two important finding of this paper not previously described in the literature. Firstly, that vitamin D levels demonstrate a significant relationship with severity of TBI and secondly, that there was a trend for QoL to be higher in patients with optimal vitamin D levels independent of severity of injury

No data regarding ethnicity or skin colour was available from the population sampled for the National Diet and Nutrition Survey. The authors have therefore assumed that patients included in this national survey sample reflect national census 2011 data regarding ethnicity with $86 \%$ of the population being of Caucasian origin.(23) Our study has a lower percentage of Caucasians at $73 \%$ and so we would expect to have a higher pre-injury prevalence of VDD, although there is no way to prove this. However, data from a Birmingham-based outpatients paper reporting on vitamin D levels are based on a more diverse population, consisting of 38\% Caucasian, 15\% Afro-Caribbean, 30\% Asian and 17\% other.(22) The authors have concluded therefore that our pre-injury baseline prevalence of VDD lies somewhere between the UK national average of $22.8 \%$ and the Birmingham average of $24 \%$.

The National Diet and Nutrition Survey 2014 demonstrates variation in vitamin D deficiency across different age groups and genders.(7) UK females aged 11-18 years appear to hold the highest percentage of vitamin D deficient individuals at $24.4 \%$ out of all groups reported. In our study, the highest prevalence of VDD is in males aged 19-64 years. This finding is most likely an epiphenomenon however, as this group also holds the highest percentage of severely injured patients who, as we have demonstrated, have significantly lower levels of vitamin D.

QoL and functional outcome are notoriously difficult to assess in TBI patients. Our results demonstrate that there is a trend for a higher quality of life in patients with optimal vitamin $D$ levels compared to those who are deficient. However, the correlation between vitamin D and QOLIBRI score is only weak. The current tools used to establish QoL and function are blunt and do not reflect more subtle changes found within higher functioning individuals. It is for this reason perhaps that no significant changes in quality of life have been demonstrated between cohorts with deficient vitamin $D$ 
and insufficient or adequate vitamin D levels. The aim of this study was not to assess functional outcome and so, such data were not collected. Any future study design - if intending to investigate functional outcome - should include detailed follow-up evaluation with a battery of assessments.

A recent retrospective study published by Jamall et al in February 2016 also analysed vitamin D in TBI patients.(24) Whilst there are plenty of similarities between that study and this, there are several key differences, which may account for our different findings. Firstly in our study we defined vitamin D deficiency in accordance with the NICE definition of vitamin D deficiency $(<25 \mathrm{nmol} / \mathrm{L})$, a cut-off that is widely used across the majority of clinical vitamin D studies.(6) Jamall et al. defined VDD as serum $25-\mathrm{OHD}<40 \mathrm{nmol} / \mathrm{L}$ and this explains why the group reported a higher prevalence of VDD in their patient cohort.

Jamall et al used several different self-reported questionnaires to measure outcome against TBI patients' vitamin D status. QoL was measured by Nottingham Health Profile and Short Form 36; no significant difference was found between vitamin D and QoL. Sleep was measured using the Epworth Sleepiness Scale and the Pittsburgh Sleep Quality Index, with neither demonstrating a relationship between vitamin D status and sleep. However, the group did demonstrate that TBI patients with VDD had worse depressive symptoms when measured using the Beck Depression Inventory II, in addition to poorer cognition, as defined by the Addenbrookes Cognitive Examination - Revised.

The Jamall et al study utilised the Mayo classification of TBI to group severity, whereas we used postresuscitative GCS to classify into mild, moderate or severe. Classifying severity using GCS identified a mild TBI category, which made up the majority of our patient cohort at $63 \%$. Our data demonstrate that although there is a statistically significant difference in VDD between mild and severe TBI, we found no significant difference between moderate and severe cohorts. It may therefore be the case that the Jamall study did not include enough patients with a milder severity of injury to detect a significant difference between their different markers of TBI severity. The conflicting findings between these two studies again highlights the need for further research into vitamin D in TBI and a thorough approach to study design.

There are several hypotheses to explain the high prevalence of VDD in TBI clinic patients. Perhaps the simplest explanation is that after a brain injury, there is often a change in behaviour. When we refer back to the risk factors for VDD identified by the NICE guidance,(6) it is clear that one large 
factor is commonplace in patients post-TBI. Those with even mild TBI may become withdrawn, suffer with depression or take time off work along with a myriad of other symptoms leading to less time spent outdoors and reduced exposure to sunlight. Patients may also undergo a change in diet, especially those who require long-term nasogastric or PEG feeding. No doubt these factors play a role in the increased VDD prevalence in the TBI population; however, recent evidence supports the multiple neurological roles of vitamin D. Could these mechanisms somehow be depleting vitamin D stores post-injury?

The role of vitamin $D$ in neurological functioning was first suggested after the discovery of high affinity vitamin D receptors (VDRs) in the pituitary, forebrain, hindbrain and spinal cord of rats.(25) In addition to this discovery the enzyme 1a-hydroxylase has also been identified in multiple areas of the human brain. Classically found in the kidney, 1a-hydroxylase converts $25-\mathrm{OHD}$ into the active vitamin D molecule $1,25(\mathrm{OH})_{2} \mathrm{D}_{3}$. The enzyme was found in the cytoplasm of glial cells and neurons, both in the presence and absence of VDRs in the nuclei. The abundance of 1a-hydroxylase within the brain suggests that, just like the kidneys, active vitamin D plays an important local role within the CNS, implying autocrine and/or paracrine function.(26)

Vitamin D has demonstrated neuroprotective effects via several different mechanisms. Many papers have reported the ability of vitamin D to alter levels of intra-cerebral neurotrophic factors (27-34), which have, in turn, been demonstrated to have both protective and restorative effects.(28)Vitamin D also has been found to reduce oxidative stress by dampening the toxic effects of free radicals and reactive oxygen species.(35-37)

Vitamin $\mathrm{D}$ has been proven to influence neuroinflammation process' by altering both amyloid and tau protein pathways $(38,39)$ and altering toll-like receptor 4 expression.(40) Vitamin D receptors have also been recognised as transcription regulation factors since the 1980 s(41) and genomic mapping has demonstrated VDR clusters in close proximity to genes coding for neurological autoimmune conditions such as MS.(42) Vitamin D also appears to play a role in neuroplasticity with upregulation of growth factors and alterations to the developing foetal brain. $(33,43,44)$

Critical illness and admission to intensive care unit (ITU) have also been extensively studied in relation to VDD. There is increasing evidence that VDD is associated with poorer outcome in ITU patients. Lower serum vitamin D levels on admission to ITU have been associated with increased 28- 
day mortality in septic patients(45) and increased length of stay.(46) This would suggest that premorbid VDD is a risk factor for poorer outcome. Our data demonstrate that patients with a severe TBI have increased rates of VDD in clinic when compared to patients with a mild TBI. Rather than the severity of TBI contributing to the development of VDD, could pre-morbid VDD be leading to prolonged ITU stays and poorer outcome? A small retrospective study conducted in 2014 demonstrated no significant correlation between admission vitamin D and neurological "outcome" on discharge from ITU in TBI patients; however, the outcome measured was the difference between admission GCS and ITU discharge GCS. The authors highlighted several issues with the study design and commented on the small sample size and recommended a well-designed prospective study.(47)

The authors of this paper put forward three hypotheses to account for the high prevalence of vitamin D deficiency in TBI clinic patients. One; behavioural changes following injury lead to decreased vitamin $\mathrm{D}$ intake by changes in diet or exposure to sunlight. Two; vitamin D stores are depleted through the neuroprotective and restorative processes occurring following brain injury. Three; VDD develops following any period of critical illness as a systemic response. There are likely elements at play from all three of these factors.

An intricate interplay may exist between TBI severity and vitamin D levels. Pre-morbid VDD may lead to a more severe head injury, as less vitamin D is available to mount an anti-neuroinflammatory, neuroprotective or neuroplastic response to injury. Conversely a more severe injury could theoretically "use-up" available stores of vitamin D leading to increased rates of deficiency. 


\section{Limitations}

The most obvious limitation of the study is that no pre-morbid vitamin $D$ status was available to establish baseline percentage of vitamin $D$ deficiency within the patient cohort. As it is impossible to perform venepuncture for research purposes prior to the patient suffering TBI, the authors advise that any future study into vitamin D in TBI aims to take biological samples as close to the time of injury as is possible. Further research, however, is required to establish over what time period post-injury vitamin D levels fall.

We did not collect any data on additional injuries many of the patients were referred to the clinic from other hospitals and clinical data were sparse. The authors recognise the limitations of categorising severity of injury by post-resuscitative GCS and advise that future prospective study categorises severity of injury by additional means such as Injury Severity Score or the New Injury Severity Score collected formally by the English and Welsh Trauma Audit Research Network (TARN).

Body mass indexes were not collected prospectively. As obesity has been identified to influence vitamin D levels we advise future study designs to include BMI measurement as a variable. 


\section{CONCLUSION}

Vitamin D deficiency is more prevalent in the TBI population compared to the UK general population and the local population of Birmingham. Patients suffering a severe TBI have significantly lower levels of vitamin $\mathrm{D}$ at follow up compared to patients suffering mild TBI. There was also a trend for QoL to be poorer in patients with deficient levels of vitamin $D$ compared to optimal levels, independent of severity of injury.

The implications of data presented in this paper and potential for future studies are vast. Firstly we must establish whether drops in vitamin D levels following injury are specific to TBI or whether this phenomenon occurs following all trauma and critical illness. Secondly, the natural history of vitamin $D$ following injury needs to be described and mapped to establish at what stage VDD develops.

We already know that treating VDD in the community reduces the risk of fractures and cardiovascular disease. It is therefore important that we highlight TBI patients as an additional at-risk group and routinely screen for the condition as we do for pituitary dysfunction. In addition, this paper has highlighted the potential use of vitamin $\mathrm{D}$ as a therapeutic agent and a randomised control trial of vitamin D therapy in TBI should be investigated. 


\section{AUTHOR DISCLOSURE STATEMENT}

The NIHR Surgical Reconstruction and Microbiology Research Centre (SRMRC) is a partnership

between The National Institute for Health Research, University Hospitals Birmingham NHS Foundation Trust, the University of Birmingham, and the Royal Centre for Defence Medicine.

This article presents independent research supported by the NIHR. The views expressed are those of the authors and not necessarily those of the NHS, the NIHR, the Department of Health, the University of Birmingham, or the Ministry of Defence.

No competing financial interests. 


\section{ACKNOWLEDGEMENTS}

The authors would like to acknowledge the research team at the SRMRC for both clinical and logistical support. 


\section{REFERENCES}

1. Maas AIR, Stocchetti N, Bullock R. Moderate and severe traumatic brain injury in adults. The Lancet Neurology. 2008;7(8):728-41.

2. Norman AW. Sunlight, season, skin pigmentation, vitamin D, and 25-hydroxyvitamin

D: integral components of the vitamin D endocrine system. The American journal of clinical nutrition. 1998;67(6):1108-10.

3. Holick MF. Vitamin D Physiology, Molecular Biology and Clinical Applications. 2 ed. New York: Humana Press; 20102010.

4. Choices N. Vitamins and minerals- vitamin D 2015 [cited 2015 15th December]. Available from: http://www.nhs.uk/Conditions/vitamins-minerals/Pages/Vitamin-D.aspx.

5. Mumsnet. Vitamin D? 2015 [cited 2015 15th December]. Available from: http://www.mumsnet.com/Talk/pregnancy/1390553-Vitamin-D.

6. National Institute for Health and Clinical Excellence. Vitamin D: increasing supplement use in at-risk groups. NICE guidelines [PH56]. 2014 November 2014. Report No.

7. Public Health England. National Diet and Nutrition Survey. Results from Years 1, 2, 3 and 4 (combined) of the Rolling Programme (2008/2009 - 2011/2012). Agency FS; 2014 2014. Report No.: Contract No.: PHE publications gateway number: 2014051.

8. MedlinePlus. Craniotabes MedlinePlus2015 [Available from: https://www.nlm.nih.gov/medlineplus/ency/article/001591.htm.

9. Dini C, Bianchi A. The potential role of vitamin D for prevention and treatment of tuberculosis and infectious diseases. Annali dell'Istituto superiore di sanita. 2012;48(3):31927. 
10. Mehta S, Hunter DJ, Mugusi FM, Spiegelman D, Manji KP, Giovannucci EL, Hertzmark E, Msamanga GI, Fawzi WW. Perinatal outcomes, including mother-to-child transmission of HIV, and child mortality and their association with maternal vitamin D status in Tanzania. The Journal of infectious diseases. 2009;200(7):1022-30.

11. Villamor E. A potential role for vitamin D on HIV infection? Nutrition reviews. 2006;64(5 Pt 1):226-33.

12. Norman PE, Powell JT. Vitamin D and cardiovascular disease. Circulation research. 2014;114(2):379-93.

13. Edlich R, Mason SS, Chase ME, Fisher AL, Gubler K, Long WB, 3rd, Giesy JD, Foley ML. Scientific documentation of the relationship of vitamin D deficiency and the development of cancer. Journal of environmental pathology, toxicology and oncology : official organ of the International Society for Environmental Toxicology and Cancer. 2009;28(2):133-41.

14. Holick MF. Vitamin D: a d-lightful solution for health. Journal of investigative medicine : the official publication of the American Federation for Clinical Research. $2011 ; 59(6): 872-80$.

15. Balvers MG, Brouwer-Brolsma EM, Endenburg S, de Groot LC, Kok FJ, Gunnewiek JK. Recommended intakes of vitamin D to optimise health, associated circulating 25hydroxyvitamin D concentrations, and dosing regimens to treat deficiency: workshop report and overview of current literature. Journal of nutritional science. 2015;4:e23.

16. Kokshoorn NE, Smit JW, Nieuwlaat WA, Tiemensma J, Bisschop PH, Groote Veldman R, Roelfsema F, Franken AA, Wassenaar MJ, Biermasz NR,Romijn JA, Pereira AM. Low prevalence of hypopituitarism after traumatic brain injury: a multicenter study. European journal of endocrinology / European Federation of Endocrine Societies. 2011;165(2):225-31.

17. Klose M, Stochholm K, Janukonyte J, Lehman Christensen L, Frystyk J, Andersen M, Laurberg P, Christiansen JS, Feldt-Rasmussen U. Prevalence of posttraumatic growth hormone deficiency is highly dependent on the diagnostic set-up: results from The Danish 
National Study on Posttraumatic Hypopituitarism. The Journal of clinical endocrinology and metabolism. 2014;99(1):101-10.

18. Boughey JC, Yost MJ, Bynoe RP. Diabetes insipidus in the head-injured patient. The American surgeon. 2004;70(6):500-3.

19. Hollo A, Clemens Z, Kamondi A, Lakatos P, Szucs A. Correction of vitamin D deficiency improves seizure control in epilepsy: a pilot study. Epilepsy \& behavior : E\&B. 2012;24(1):131-3.

20. QOLIBRI. What is QOLIBRI? 2015 [cited 2015 11th December]. Available from: http://www.qolibrinet.com/.

21. Scientific Advisory Committe on Nutrition. Draft Vitamin D and Health report. Scientific consultation: 22 July to 23 September 2015. SACN, Health Do; 2015 2015. Report No.

22. Ford L, Graham V, Wall A, Berg J. Vitamin D concentrations in an UK inner-city multicultural outpatient population. Annals of clinical biochemistry. 2006;43(Pt 6):468-73.

23. Office of National Statistics. Ethnicity and National Identity in England and Wales: 2011: Office for National Statistics; 2011 [cited 2016 11th June]. Available from: http://www.ons.gov.uk/peoplepopulationandcommunity/culturalidentity/ethnicity/articles/ethni cityandnationalidentityinenglandandwales/2012-12-11.

24. Jamall OA, Feeney C, Zaw-Linn J, Malik A, Niemi ME, Tenorio-Jimenez C, Ham TE, Jilka SR, Jenkins PO, Scott G, Li LM,Gorgoraptis N, Baxter D, Sharp DJ, Goldstone AP. Prevalence and Correlates of Vitamin D Deficiency in Adults after Traumatic Brain Injury. Clinical endocrinology. 2016.

25. DeLuca GC, Kimball SM, Kolasinski J, Ramagopalan SV, Ebers GC. Review: The role of vitamin D in nervous system health and disease. Neuropathology and Applied Neurobiology. 2013;39(5):458-84. 
26. Eyles DW, Smith S, Kinobe R, Hewison M, McGrath JJ. Distribution of the vitamin D receptor and 1 alpha-hydroxylase in human brain. Journal of chemical neuroanatomy. $2005 ; 29(1): 21-30$.

27. Wang Y, Chiang YH, Su TP, Hayashi T, Morales M, Hoffer BJ, Linz SZ. Vitamin D(3) attenuates cortical infarction induced by middle cerebral arterial ligation in rats. Neuropharmacology. 2000;39(5):873-80.

28. Minnich JE, Mann SL, Stock M, Stolzenbach KA, Mortell BM, Soderstrom KE, Bohn MC, Kozlowski DA. Glial cell line-derived neurotrophic factor (GDNF) gene delivery protects cortical neurons from dying following a traumatic brain injury. Restorative neurology and neuroscience. 2010;28(3):293-309.

29. Pardridge WM, Boado RJ. Pharmacokinetics and safety in rhesus monkeys of a monoclonal antibody-GDNF fusion protein for targeted blood-brain barrier delivery. Pharmaceutical research. 2009;26(10):2227-36.

30. Camu W, Tremblier B, Plassot C, Alphandery S, Salsac C, Pageot N, Juntas-Morales R, Scamps F, Daures JP, Raoul C. Vitamin D confers protection to motoneurons and is a prognostic factor of amyotrophic lateral sclerosis. Neurobiology of aging. 2014;35(5):1198205.

31. Hawes JE, Tesic D, Whitehouse AJ, Zosky GR, Smith JT, Wyrwoll CS. Maternal vitamin $\mathrm{D}$ deficiency alters fetal brain development in the BALB/c mouse. Behavioural brain research. 2015;286:192-200.

32. Orme RP, Bhangal MS, Fricker RA. Calcitriol imparts neuroprotection in vitro to midbrain dopaminergic neurons by upregulating GDNF expression. PloS one. 2013;8(4):e62040.

33. Eyles D, Brown J, Mackay-Sim A, McGrath J, Feron F. Vitamin D3 and brain development. Neuroscience. 2003;118(3):641-53. 
34. Shirazi HA, Rasouli J, Ciric B, Rostami A, Zhang GX. 1,25-Dihydroxyvitamin D3 enhances neural stem cell proliferation and oligodendrocyte differentiation. Experimental and molecular pathology. 2015;98(2):240-5.

35. Smith MP, Fletcher-Turner A, Yurek DM, Cass WA. Calcitriol protection against dopamine loss induced by intracerebroventricular administration of 6-hydroxydopamine. Neurochemical research. 2006;31(4):533-9.

36. Wang JY, Wu JN, Cherng TL, Hoffer BJ, Chen HH, Borlongan CV, Wang Y. Vitamin $\mathrm{D}(3)$ attenuates 6-hydroxydopamine-induced neurotoxicity in rats. Brain research. $2001 ; 904(1): 67-75$.

37. Ibi M, Sawada H, Nakanishi M, Kume T, Katsuki H, Kaneko S, Shimohama S, Akaike A. Protective effects of 1 alpha,25-(OH)(2)D(3) against the neurotoxicity of glutamate and reactive oxygen species in mesencephalic culture. Neuropharmacology. 2001;40(6):761-71.

38. Briones TL, Darwish $H$. Decrease in age-related tau hyperphosphorylation and cognitive improvement following vitamin $D$ supplementation are associated with modulation of brain energy metabolism and redox state. Neuroscience. 2014;262:143-55.

39. Briones TL, Darwish H. Vitamin D mitigates age-related cognitive decline through the modulation of pro-inflammatory state and decrease in amyloid burden. Journal of neuroinflammation. 2012;9:244.

40. Tang H, Hua F, Wang J, Yousuf S, Atif F, Sayeed I, Stein DG. Progesterone and vitamin $\mathrm{D}$ combination therapy modulates inflammatory response after traumatic brain injury. Brain injury. 2015:1-10.

41. Baker AR, McDonnell DP, Hughes M, Crisp TM, Mangelsdorf DJ, Haussler MR, Pike JW, Shine J, O'Malley BW. Cloning and expression of full-length cDNA encoding human vitamin D receptor. Proceedings of the National Academy of Sciences of the United States of America. 1988;85(10):3294-8. 
42. Ramagopalan SV, Heger A, Berlanga AJ, Maugeri NJ, Lincoln MR, Burrell A, Handunnetthi L, Handel AE, Disanto G, Orton SM, Watson CT,Morahan JM, Giovannoni G, Ponting CP, Ebers GC, Knight JC. A ChIP-seq defined genome-wide map of vitamin D receptor binding: associations with disease and evolution. Genome research. 2010;20(10):1352-60.

43. Wion D, Macgrogan D, Neveu I, Jehan F, Houlgatte R, Brachet P. 1,25Dihydroxyvitamin D3 is a potent inducer of nerve growth factor synthesis. Journal of Neuroscience Research. 1991;28(1):110-4.

44. Neveu I, Naveilhan P, Jehan F, Baudet C, Wion D, De Luca HF, Brachet P. 1,25dihydroxyvitamin D3 regulates the synthesis of nerve growth factor in primary cultures of glial cells. Brain research Molecular brain research. 1994;24(1-4):70-6.

45. Chen Z, Luo Z, Zhao X, Chen Q, Hu J, Qin H, Liang X, Suo Y. Association of vitamin D status of septic patients in intensive care units with altered procalcitonin levels and mortality. The Journal of clinical endocrinology and metabolism. 2015;100(2):516-23.

46. Alizadeh $N$, Khalili $H$, Mohammadi $M$, Abdollahi A. Serum Vitamin D levels at admission predict the length of intensive care unit stay but not in-hospital mortality of critically ill surgical patients. Journal of research in pharmacy practice. 2015;4(4):193-8.

47. Socci FI, Di Valvasone S, Cecchi A, Bonizzoli M, Terreni A, Peris A. Vitamin D level could affect the recovery rate in traumatic brain injury: a retrospective study. Critical Care. 2014;18(Suppl 1):P471-P. 